\title{
Protein Arginine Methyltransferase 8: Tetrameric Structure and Protein
}

Substrate Specificity

Wei-Chao Lee, $\uparrow$ Wen-Ling Lin, $\uparrow$ Tsutomu Matsui, $\uparrow$ Eric S.-W. Chen, $\uparrow$ Tong-You Wade Wei, $\uparrow$ Wen-Hsuan Lin, $\uparrow$ Hao Hu, $\S$ Yujun George Zheng, $§$ Ming-Daw Tsai, $\uparrow$ and Meng-Chiao Ho*, $\dagger$

\section{Supplemental Information - Materials and Methods}

MS analysis for in vitro methylation of NIFK- The recombinant methylated NIFK was separated from tPRMT8 by electrophoresis followed by in-gel digestion with Lys-C (Wako. Chemicals USA, VA). Digested peptides were extracted and dried. Peptide samples were reconstituted in $0.1 \%$ FA and analyzed on Waters Synapt G2 HDMS (Waters, Milford, MA). The C18 column was maintained at $35{ }^{\circ} \mathrm{C}$ and the bound peptides were eluted with a gradient of 5-35\% of CAN in the presence of $0.1 \%$ FA. Data acquisition was performed using data directed analysis (DDA) method. The DDA method included one full MS scan (m/z 350-1600, $1 \mathrm{~s} / \mathrm{scan})$ and three MS/MS scans (m/z 100-2000, $1 \mathrm{~s} / \mathrm{scan})$. Analysis of all MS/MS samples was performed using Mascot (Matrix Science; version 2.4.0). Mascot was set up to search the Swiss-Prot human database. Database search against Mascot was performed with a fragment ion mass tolerance of $0.05 \mathrm{Da}$ and a parent ion tolerance of $25 \mathrm{ppm}$. Two missed cleavages were allowed during trypsin digestion. Oxidation (Met), carbamidomethyl (Cys) and arginine methylation (methyl and dimethyl) were specified as variable modification.

\section{Circular dichroism analysis}

Both TPRMT8 and tPRMT8C were purified as described and samples were prepared to the desired concentration of $\sim 10 \mu \mathrm{M}$ in $50 \mathrm{mM}$ sodium phosphate, $\mathrm{pH} 7.5,100 \mathrm{mM}$ sodium chloride. All CD spectra were collected with an Aviv CD 202 spectrometer (Lakewood, $\mathrm{NJ}$ ) at $25^{\circ} \mathrm{C}$. The samples were placed in a $1 \mathrm{~mm}$ path length cuvette for CD spectra from 190 to $260 \mathrm{~nm}$ wavelength of step of $0.5 \mathrm{~nm}$. For thermal denaturation experiments, the sample was monitored at $215 \mathrm{~nm}$ from 25 to $95{ }^{\circ} \mathrm{C}$ with a $2{ }^{\circ} \mathrm{C}$ interval. The data curves were analyzed using SigmaPlot 8.02 (SPSS Inc.). 